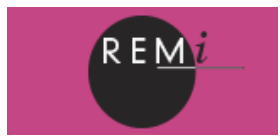

Revue européenne des migrations internationales

vol. 22 - n² | 2006

$20^{\mathrm{e}}$ anniversaire

\title{
Migrations, la spatialisation du regard
}

\section{Gildas Simon}

\section{(2) OpenEdition}

\section{Journals}

Édition électronique

URL : https://journals.openedition.org/remi/2815

DOI : $10.4000 /$ remi.2815

ISSN : $1777-5418$

Éditeur

Université de Poitiers

\section{Édition imprimée}

Date de publication : 1 juillet 2006

Pagination : 9-21

ISBN : 2-911627-42-3

ISSN : 0765-0752

Référence électronique

Gildas Simon, « Migrations, la spatialisation du regard », Revue européenne des migrations internationales [En ligne], vol. 22 - n² | 2006, mis en ligne le 01 juin 2009, consulté le 14 avril 2022

URL : http://journals.openedition.org/remi/2815 ; DOI : https://doi.org/10.4000/remi.2815

Ce document a été généré automatiquement le 14 avril 2022.

(c) Université de Poitiers 


\title{
Migrations, la spatialisation du regard
}

\author{
Gildas Simon
}

1 Le domaine de la recherche sur les migrations internationales a connu une véritable mutation au cours des trente dernières années: développement et dans une certaine mesure banalisation de l'interdisciplinarité et plus encore de la transdisciplinarité, multiplication des formations à la recherche, des séminaires, des rencontres et des colloques, lancement de revues nouvelles en Europe, en Amérique latine et en Asie, multiplication phénoménale de la production et des publications sur les différents types de supports (édition traditionnelle, internet). Étonnante métamorphose du champ qui ne peut que susciter toutes sortes de questionnements et d'interprétations car si on peut y lire l'expression des changements structurels migratoires eux-mêmes, les mutations de " paysages migratoires » et de "systèmes migratoires » de plus en plus complexes, l'évolution des représentations, l'importance voire la gravité des enjeux matériels et symboliques portés par les mobilités actuelles, nous pouvons y percevoir aussi, me semble-t-il, le signe chargé de sens du renouvellement des approches et du regard porté, de l'intégration de nouveaux outils, d'autres modes d'analyse, en bref de tout ce qui a rendu possible l'ouverture et l'attraction réelle de ce champ scientifique sur de nouvelles générations de chercheurs.

\section{Spatialisation des approches}

2 Dans ce changement en profondeur du regard porté, les questions d'espace, de spatialité, de territorialité ont pris une place croissante, qu'il s'agisse évidemment du rapport des migrants aux lieux et aux espaces matériels, sociaux, politiques ou idéels reliés par ces migrations, mais aussi des pratiques sociales développées à travers des espaces transnationaux, de l'architecture des constructions identitaires à différentes échelles ou encore des espaces normatifs ou réglementaires, dont «l'espace Schengen » offre un exemple particulièrement illustratif. La spatialisation croissante des modes d'analyse à l'intérieur du champ scientifique migratoire s'est accompagnée d'une 
véritable floraison de termes et de notions spatialement référées, particulièrement révélatrices de ce nouveau "besoin de spatialité ». Les chercheurs disposent aujourd'hui de toutes sortes d'" espèces d'espaces ", selon une formule empruntée à Georges Perec, pour penser les migrations et l'ensemble des mobilités: espaces migratoires proprement dits, champs migratoires (campos migratorios, migration fields), territoires migratoires, espaces circulatoires, territoires circulatoires, territoires de la mobilité, espaces transnationaux (transnational fields). L'analyse des fonctionnements, quant à elle, fait désormais appel à des notions de dynamiques spatiales, d'interactions spatiales et d'interspatialité, de cospatialité, de ressources spatiales et de capital spatial, et bien entendu, de territorialisation, déterritorialisation, reterritorialisation, etc.

3 Cette imprégnation spatiale du champ de la recherche migratoire apparaît concrètement à travers l'ensemble des articles et notes de recherche publiés ici même, par la REMI depuis son lancement en 1985, approche évidemment prismatique, mais un numéro anniversaire est une bonne occasion de pratiquer ce type d'exercice rétrospectif. On la retrouve tant dans les choix éditoriaux (numéros à thème et dossiers centrés) que dans le contenu même des articles proposés, dans le traitement réalisé par des auteurs venus d'horizons disciplinaires et géographiques multivariés... Cette prégnance qui dépasse le souci ordinaire de localisation ou de distribution de populations pourrait n'exprimer qu'un banal phénomène de sélection spatiale, consciente ou inconsciente, des articles et de leur affichage sur ce registre par des comités de lecture et de rédaction où, pour des raisons liées à l'histoire même de la REMI, géographes et géographie sont largement représentés, mais des sondages dans des revues aux mêmes exigences scientifiques aboutiraient probablement à des constats assez voisins.

4 Nous le savons bien, l'espace a investi le monde de nos représentations à quelque échelle que ce soit, depuis « l'espace d'intimité » jusqu'à celui de la mondialisation et de la mondialité; il est devenu l'une des catégories efficaces pour penser «l'être-aumonde ", décalant et subvertissant au passage la référence fondamentale au temps et à la durée qui avait constitué l'un des fils d'acier des cultures précédentes. Alain Touraine dans l'un des articles introductifs à «La Planète nomade» (Knafou dir, 1998), ouvrage issu du Festival International de Saint-Dié, observait que «les catégories de l'espace tendent à remplacer dans notre expérience les catégories du temps, ou tout au moins, à devenir plus importantes qu'elles ». La réflexion, l'agir, mais aussi le ressenti de notre monde se réalisent aujourd'hui sur le mode de la spatialisation, à quelque échelle que ce soit, incitant au renouvellement de la réflexion sur les implications de ce «nouveau» mode de penser toutes sortes de questions, comme, par exemple, la pauvreté et les politiques sociales urbaines (Tissot et Poupeau, 2005).

5 Il n'est, certes, pas facile d'analyser les composantes de cette imprégnation spatialisante par rapport aux modes d'analyse précédents, de déchiffrer le sens profond de cette évolution, on le ressent et on le devine plus qu'on ne le démontre dans bon nombre de travaux des sciences sociales (sociologie, anthropologie, ethnologie, politologie); une certitude, cependant, le regard général sur le monde, avec ses dynamiques en réseaux et ses modes de fonctionnement à différentes échelles, change en profondeur avec l'appropriation de ce mode d'analyse des sociétés, avec le passage par cette "porte d'entrée " considéré longtemps comme le territoire exclusif des géographes (Lévy, 1999). Pour ceux-ci aussi, ce nouveau mode de questionnement du 
monde sur lui-même, mais aussi l'apport des autres sciences sociales dans leur champ de prédilection, la transversalité ont des implications importantes, car ils entraînent la remise en cause de leurs propres schémas disciplinaires et posent une exigence théorique qui faisait trop souvent défaut. L'interrogation sur les nouveaux modes de spatialisation du regard enveloppe désormais le cours de toute réflexion géographique, ainsi que l'exprimait tout récemment l'introduction à un séminaire au titre explicite Penser l'espace (Lausanne juillet 2005) : «ne sommes - nous pas face à un changement de modes de spatialisation des sociétés humaines, qui, moins soumises à l'emprise territoriale, se métissent de réticularités multiples? De telles mutations nécessitent de développer des concepts, théories et modèles qui soient capables d'appréhender ce contexte en mutation ».

6 Domaine emblématique s'il en est du bouleversement, celui de la mobilité spatiale, et plus spécifiquement des migrations internationales est au cœur de l'ensemble des questionnements posés par le bouleversement des sociétés, ce "Grand dérangement " où par le recours à la métaphore migratoire (exode forcé des Acadiens après le traité de 1759) Georges Balandier pointe avec justesse la valeur symbolique de la mobilité, de toutes les formes de mobilité dans l'incertitude et le trouble des mutations en cours. "(Le nouveau monde) est aujourd'hui planétaire et s'accomplit dans le temps, il n'impose pas une coupure territoriale mais une séparation d'avec le passé, la période historique achevée dans la seconde moitié du siècle dernier. Il fait de tous les contemporains, partout, des façons d'immigrés dans le temps, des gens qui se découvrent en partie étrangers au monde qu'ils font pourtant... Si la métaphore du Grand Dérangement est pertinente, elle signale à la fois le grand déplacement dans ce qui substitue le territoire et l'astreinte à un nouveau commencement de l'aventure (post) humaine » (Balandier, 2005). Effectivement, plus que la figure de l'étranger qui est de toutes les périodes, celle du migrant avec ou sans papiers, du circulant transfrontières, signe bien les paradoxes et les contradictions de la mondialisation actuelle, entre un immense désir de mobilité, l'abolition systématique des obstacles et des contraintes à la circulation universelle des flux, et l'assignation à résidence appliquée aux habitants des pays pauvres de la planète; en contrepoint de l'ouverture et de la connexion irrésistible des espaces physiques et idéels, le protectionnisme qui s'installe n'a guère d'équivalent dans l'histoire des migrations de l'humanité.

Questionnements territoriaux et interrogations identitaires brassent en profondeur ce monde de la mobilité globale et des migrations car les migrations sont lieux de production toujours renouvelés, de représentations, de mythes, de cultures et d'identités nouvelles impensées, source d'imaginaires de toutes sortes, matrices densément chargées d'affects. Les interférences entre les réalités matérielles, la puissance de l'imaginaire tant dans les sociétés d'origine que dans les pays d'installation, la force des affects fonctionnant à travers les espaces connectés par la migration sont telles que dans la majorité des États les politiques migratoires se trouvent renvoyées, de facto, au statut d'illusion réglementaire ou de discours à finalité électorale. L'ampleur des transferts de revenus migratoires (plus de 250 milliards de dollars en 2004; deuxième rang mondial après les transactions liées aux hydrocarbures), la géographie des circuits financiers qui l'exprime, signent l'importance de cette réalité affective autant qu'économique qui s'est inscrite au cœur de l'espace planétaire. Le champ scientifique ne pouvait échapper à ce renouvellement des questionnements d'ensemble. 


\section{L'approche intégrée de l'espace migratoire}

8 On sait que parmi les insatisfactions théoriques et les limites de l'approche habituelle des faits migratoires, il y avait, et il y a toujours, la dichotomie de l'analyse spatiale, l'émigration d'un côté, l'immigration de l'autre, mais aussi l'unilatéralité de la démarche privilégiant d'ailleurs beaucoup plus fréquemment le point de vue du pays d'arrivée par rapport au lieu et au pays de départ, (modes de pensée - il faut le dire quela dépendance vis-à-vis des modes de recensements et d'enregistrements statistiques et le difficile raccordement des différentes sources nationales ont eu tendance à rigidifier). Cette vision induisant une segmentation implicite et rigide de l'espace effectivement concerné a fait place progressivement à une approche souple des mobilités où des identités territoriales se déploient dans des contextes de multilocalisation et de multipolarité, dans des espaces de vie trans-frontières à significations diverses où des dynamiques sans cesse renouvelées articulent des lieux de départ, d'implantations successives mais aussi de transits éphémères ou prolongés.

Mais le cheminement vers une prise en compte de la globalité de l'espace concerné estil aussi "post-moderne" et doit-on dire aussi nord-américain, que le voient aujourd'hui certains auteurs à la recherche d'une théorie spatiale satisfaisante des migrations et de la mobilité générale? Les chemins à la recherche des sorties des frontières mentales qui cloisonnaient, segmentaient et amputaient l'analyse de ce champ social, les itinéraires et les parcours des chercheurs dans les différentes disciplines, ont été longs et complexes; me vient à l'esprit la métaphore (inévitablement naturaliste...) du réseau anastomosé du cours d'un fleuve aux multiples bras, tantôt semblant s'éloigner, tantôt se rejoignant pour s'écarter à nouveau, avant de se rejoindre. Pour ma part, j'y verrais plutôt la convergence, depuis les années soixante-dix, d'une double dynamique, inscrite du côté nord-américain dans des logiques plutôt de type systémique: rapports villes-campagnes (Mabogunje, 1970), systèmes migratoires (Massey et al., 1993), transnationalisme (Portes, 1999) et du côté européen, dans des approches peut-être un peu plus «spatialisantes" autour notamment des notions de champs migratoire, d'espaces migratoires, puis de territoires circulatoires, de territoires de la mobilité. Citons le travail pionnier du géographe suédois T. Hagërstrand et de l'École de Lund (1957), de géographes français, R. Schwab, R. Béteille (1974), de sociologues portugais, Castro da Alméida (1974), nos premiers travaux sur l'espace migratoire (1976), puis sur le champ migratoire international (1979). Ce dernier concept dont la mise en œuvre méthodologique fut laborieuse, car obligeant à casser les cadres mentaux de l'analyse traditionnelle, ne suffirait plus aujourd'hui à rendre compte de ces réalités transnationales mouvantes, mais il me semble - avec le recul des années et la conscience de la difficulté d'apprécier son propre apport dans une évolution intellectuelle - que cette première application du concept de champ migratoire à une migration internationale, dans le cadre de la géographie d'expression française par son approche globale, transfrontières, de l'espace migratoire d'un groupe national, d'un " collectif migrant ", contribua à ouvrir un chemin que la création par le CNRS de l'équipe Migrinter en 1985, sur la proposition du regard global et inversé « migrations internationales et sociétés d'origine ", permit de densifier, d'approfondir, d'élargir et de prolonger dans d'autres directions. 
10 Tout au long de ces années, la problématique de ces espaces sociaux inter-nationaux, transnationaux, s'est considérablement enrichie et diversifiée sur le plan théorique directement et indirectement par les travaux de sociologues et d'anthropologues. Notons cependant au passage que la conception de Pierre Bourdieu qui nous a beaucoup apporté sur le plan de la théorie du champ dans les sciences sociales, sur l'espace social, le jeu des acteurs, les enjeux masqués ou symboliques, est restée plus attachée à la figure du migrant, de l'immigré-émigré qu'à celle de l'espace et du champ migratoire et que son regard sur les migrations semble plus marqué par la vision de la rupture, du déracinement et de l'exil plus que par celle du lien et de la relation maintenus à travers l'espace et la durée. De manière plus précise sur le plan migratoire, l'apport de travaux des anthropologues du mouvement et notamment d'Alain Tarrius définissant le nouveau concept de "territoire circulatoire" sur la base de nouvelles formes de mobilité produites par des acteurs économiques particulièrement efficaces dans le champ de la mondialisation, a été particulièrement fécond et a contribué à donner une réelle épaisseur sociologique aux concepts spatiaux déjà en circulation scientifique. Avec l'ensemble des travaux sur la circulation migratoire menés autour du laboratoire Migrinter à partir du milieu des années quatre-vingt, la réappropriation du concept de diaspora, la multiplication des travaux sur les réseaux sociaux transnationaux, il est clair qu'on ne peut plus penser dans les mêmes termes la question de la spatialité migratoire et circulatoire.

11 Au sein même de la géographie, ce courant de recherche, désormais bien identifié et reconnu, ne trouve pas - faut-il le dire ? - sa complétude en lui-même, dans l'étude en boucle de l'espace pour l'espace; il s'installe d'abord dans l'idée que l'espace global de la migration internationale nous semble constituer un lieu, un observatoire pertinent de ce "phénomène social total ", ou du moins complexe et si révélateur des mutations actuelles. La densification de l'objet apportée par le concept d'espace porteur d'« un principe de réalité sociétale " (Levy, Lussault, 2003), « construction surtout collective mais accomplie aussi par une myriade d'interventions privées qui ne respectent pas toutes, immuablement, les mêmes règles et les mêmes principes d'action » (Di Méo, Buléon, 2005), l'enrichissement d'une notion reconnue comme champ symbolique autant qu' instance dynamique et causale», trouve dans l'étude des espaces migratoires, lato sensu, un champ de déploiement de l'analyse particulièrement riche et producteur de sens, qu'il s'agisse, par exemple, du passage d'une culture de la mobilité à «un capital social du mouvement» (L. Faret, 2003), de la mobilisation des « ressources spatiales » dans le cadre de constructions diasporiques (E. Ma Mung, 1999; M. Bruneau, 2004), ou de problématiques plus centrées sur des espaces soumis à des tensions de haute intensité comme le Moyen-Orient ou l'Afrique de l'Ouest.

12 La prise en compte spatialisée, globalisée traversant les frontières, le passage de l'approche segmentée à l'approche globale, constituent, me semble-t-il l'une des ruptures épistémologiques accomplies dans l'analyse contemporaine des migrations internationales. La panoplie des termes et des notions actuelles qui intègrent l'ensemble du champ parcouru par les migrants, au-delà des frontières, permet d'englober l'ensemble des lieux et des espaces de vie, non comme une succession d'espaces aux frontières délimitées, mais comme une pluralité de champs à la fois autonomes et articulés, où le rôle des acteurs sociaux apparaît plus efficace dans la dynamique migratoire réelle que celui des États et des acteurs institutionnels classiques. Cette approche spatialisée et globale qui pourrait apparaître comme le plus 
petit commun dénominateur de ces différents termes, notions, ou concepts, représente quand même un grand pas sur les chemins invisibles de la transdisciplinarité... comme le prouve l'espace même de cette revue où presque toutes les sciences sociales se retrouvent et dialoguent autour d'approches et de langages partagés par les autres. L'évolution de la perception dans ce domaine est considérable, du moins, dans le champ de la recherche, car si l'on s'en tient au discours social et politique ambiant, le décalage des représentations demeure considérable, lorsqu'on constate que la société politique, elle-même en retard sur l'évolution mentale des sociétés civiles, continue à ne penser l'ensemble de ces questions qu'en termes d'immigration et, au mieux, de codéveloppement!

\section{Territoire circulatoire ou champ migratoire?}

13 Passons rapidement sur l'espace migratoire dont l'emploi s'est largement répandu et banalisé, y compris dans le langage plus convenu des organisations internationales spécialisées. Le terme est relativement commode et présente le double avantage d'englober l'ensemble de l'espace pratiqué par les migrants (espace d'origine, d'implantation mais aussi de transit, de rebondissement), fonctionnant comme un " continuum » à différentes échelles (régionale, intercontinentale, planétaire), et de rejoindre le champ de la perception globale et immédiate qu'en ont généralement les acteurs migrants eux-mêmes, celle de l'intérieur d'un vaste "espace de vie » tendu entre l'espace d'origine et l'espace d'implantation, espace souvent discontinu physiquement mais uni affectivement et symboliquement par ou dans une sorte de tension permanente entre ici et là-bas.

14 Certes, ce terme ouvre déjà la voie à l'analyse de modes de fonctionnement territorialisés et multi-localisés, à la connaissance des pratiques migratoires de l'espace et de leurs logiques, tout en dégageant par l'analyse comparative la possibilité de recherche de spécificités de l'espace de vie pratiqué par des groupes à différents niveaux d'analyse (par exemple famille élargie, groupe villageois, ethnico-régional, national, etc.). Comme tout espace socialement produit et constitué, son fonctionnement implique un ensemble d'acteurs avec leurs logiques propres, leurs modes de fonctionnement, leurs pratiques élémentaires ou complexes; espace de représentations individuelles et collectives, il peut être chargé de sens et servir de support à toutes sortes d'interprétations.

15 Mais cette désignation très englobante présente aussi les inconvénients d'une notion descriptive, aux contours flous et aux signifiants en définitive plutôt limités; on peut la considérer comme le premier degré d'analyse mais il est nécessaire, si on veut s'engager d'avancer dans l'appréhension d'un système spatial et temporel dynamique et de formations sociales plus complexes, de recourir à un registre de termes et de notions à la fois plus diversifié et plus précis. Dans l'organisation de l'ordre des spatialités liées à la mobilité en général et à la complexification des migrations internationales en particulier, le territoire circulatoire et le champ migratoire international ou transnational se situent ou constituent un deuxième niveau d'analyse nettement plus intéressant car plus producteur de sens dans l'architecture vivante et mouvante de la planète migratoire.

16 Concept devenu fondamental dans l'analyse migratoire car la relation, l'échange, la circulation entre les différents lieux articulés par la migration internationale, pratiqués 
et connectés sur toutes sortes de plans par les migrants et leurs proches, la circularité aussi, sont constitutifs des champs et des systèmes migratoires et de leur fonctionnement intégratif; le terme de migration circulaire, est utilisé depuis longtemps en Afrique de l'Ouest pour caractériser les migrations en boucle entre différents États. Le concept circulatoire a fait florès depuis une vingtaine d'années, sans doute parce qu'il répond bien à une demande de prise en compte empirique et théorique de globalisation et de fluidité, d'articulation des différentes formes de mobilités à toutes les échelles, du local au global. Le concept de «territoires circulatoires » transfrontières a pris, à juste titre, une importance croissante dans le domaine scientifique, libérant de nouvelles capacités d'analyse dans le champ social et symbolique des sociétés concernées, notamment autour de l'émergence de nouvelles logiques du mouvement, des pratiques spatiales, sociales et culturelles qui leur sont associées. "Tout espace - écrit Alain Tarrius - est circulatoire; en revanche, tout espace n'est pas territoire. La notion de territoire circulatoire constate une certaine socialisation des espaces supports aux déplacements. Les individus se reconnaissent à l'intérieur des espaces qu'ils investissent ou traversent au cours d'une histoire commune de la mobilité, initiatrice d'un lien social original... Cette notion introduit donc une double rupture dans les acceptions communes du territoire et de la circulation. En premier lieu, elle suggère que l'ordre des sédentarités n'est pas essentiel à la manifestation du territoire; les normes ainsi produites défont les oppositions entre hiérarchies identitaires tributaires des préséances sédentaires, des références au « lieu » : exit le marquage le plus usuel entre autochtone et étranger, ethnique parfois; en somme, tous ethniques et tous autochtones de moments et de lieux, donnés à voir et à vivre en même temps. La mobilité spatiale exprime donc plus qu'un mode d'usage des espaces, le déplacement d'un lieu d'activité à un autre, mais aussi des hiérarchies sociales, des reconnaissances qui donnent force et pouvoir, qui dissimulent aux yeux des sociétés de sédentaire des violences et des exploitations non moins radicales, mais autres, parce que l'ailleurs de l'étrange se confond avec l'ailleurs du lieu pour celui demeuré dans l'immobilité de ses certitudes indigènes » (Tarrius, 1989).

Cependant - et sans renier les recherches initiées et poursuivies à Migrinter sur le thème circulatoire - nous ne partageons pas, pour notre part, le schéma qui paraît désormais admis, du moins dans le champ de la recherche, du remplacement définitif $\mathrm{du}$ paradigme migratoire par le paradigme circulatoire ou mobilitaire, voire " motilitaire "; les observations faites à l'occasion de deux colloques récents, affichés sur la circulation migratoire (Paris 2004 et Toulouse 2005), mais où la majorité des communications et des débats portaient en réalité sur les questions strictement migratoires, conforte l'idée qu'il est temps de poser explicitement la question de la pertinence de la substitution totale de l'un par l'autre et ce, par le recours à la question centrale de l'espace.

\section{Le champ migratoire : un terme encore producteur de sens?}

18 Les termes de champ migratoire et de territoire circulatoire sont-ils aussi antagonistes qu'il le parait? Entre les deux, ne s'agit-il pas plutôt de différences de degré, de déplacement du centre de gravité de l'analyse ; chacune de ces deux notions porte me semble t'il - une charge sémantique différente et complémentaire, chacune est 
porteuse de sens. L'intérêt scientifique des termes que nous utilisons, leur pertinence résident dans leur aptitude à rendre compte des réalités observées mais aussi dans leur capacité évocatoire.

Si la notion de territoire circulatoire fait référence à l'appropriation de l'espace, des lieux parcourus, reconnus par l'acteur - migrant dans un jeu de correspondances spatiales et symboliques complexes, en ce sens on dira avec Alain Tarrius que le territoire circulatoire est "producteur de normes entre ici et là-bas ", celle de champ migratoire déplace, me semble-t-il, le regard vers la structuration interne de l'espace social construit par les acteurs, sur des distances parfois considérables entre les lieux d'origine, de transit et d'installation, en faisant jouer sémantiquement la fonction générative et tensionnelle de toute notion de champ. De même qu'on dit d'une installation ou d'un circuit électriques qu'ils sont "sous tension" - le champ migratoire est un espace sous tension car investi d'une charge symbolique forte, porteuse pour le migrant d'espoirs, d'utopies ou de mythes, comme d'ailleurs aussi pour les sociétés où se sont inscrits ces champs sociaux constitués d'imaginaires profondément enracinés dans les mentalités collectives, aux frontières mouvantes de l'identité et de l'altérité. « Une collectivité humaine s'organise seulement lorsqu'elle est organisée par un mythe, par un champ symbolique qui se manifeste d'une manière efficace ». Le champ migratoire me semble demeurer l'un de ces champs symboliques où la force des représentations et du mythe est capable de structurer en profondeur l'architecture d'un espace social transnational.

C'est donc la mise sous tension qui assure l'existence, l'unité de ce champ de forces, sa capacité à transcender les distances, les frontières et qui dépasse la fonctionnalité du territoire circulatoire; le concept migratoire porte toujours une charge qui dépasse l'idée de la combinatoire de territoires associés par la migration; dans l'ordre des spatialités, il est porteur d'une densité, d'une charge tensionnelle qui apporte un élément important, essentiel dans la dynamique profonde de la pensée d'un espace de mobilité, celle d'un espace structuré sur le plan spatial, économique, culturel, affectif par un ensemble de dynamiques. « Migrations - écrivait Maurice Halbwachs en 1970 dans la mesure où les hommes qui se déplacent sont pris dans un courant, qui est un courant de l'espace mais qui est aussi un courant social. Formations sociales éphémères? Même déracinée en partie, cette population hésiterait à se mettre en marche, si elle n'obéissait pas à des forces collectives qui la dépassent ».

Le migrant est à la fois agi et agissant, jouet de forces qui le dépassent et acteur des luttes dont il est partie prenante. Les assauts tragiques d'Africains sur les barrages de la frontière de l'Union Européenne entre Maroc et Espagne témoignent, pour le moins, de l'intensité des tensions régnant à l'intérieur de certains champs migratoires, de l'importance des enjeux spatiaux, matériels et symboliques qui lui sont attachés: première entrée physique sur l'espace espéré; enjeux de l'acquisition d'un statut juridique qui rendra possible la circulation transfrontière, accès à ce qui deviendra alors, mais alors seulement, un véritable territoire circulatoire ; enjeux du choix du lieu principal de vie, de la double résidence, etc. Enjeux symboliques forts, comme le montre le débat autour de l'actuel projet de loi sur l'immigration en France, tant les sociétés d'origine comme les sociétés d'installation ne semblent pas encore prêtes à accepter le «nouveau monde» de la mobilité; enjeux existentiels enfin liés à la symbolique fondamentale de la migration, du passage de la vie et de la mort, telles que fixées dans la majorité des cultures. 

en France (1979), observant que « loin d'être un espace éphémère, mal défini, parcouru de manière anarchique par une main-d'œuvre instable, le territoire pratiqué par les travailleurs tunisiens en France apparaissait, au contraire, comme un champ migratoirebien délimité et déjà stabilisé », nous formulions l'hypothèse de la stabilité de cette forme d'organisation à travers l'espace, de sa résistance au temps. «La relève de la première génération de migrants par la seconde, celle des enfants d'immigrés dont la jeunesse s'inscrit désormais dans les cadres des grandes villes françaises, peut remettre en cause le maintien de ce champ social international. En effet les processus d'« acculturation » au sein de ce groupe en France, plus que son intégration véritable à l'intérieur de la société française, risquent de détendre, de dissoudre peu à peu tous les liens personnels et collectifs, qui attachent les émigrés de la première génération à leurs communautés d'origine par l'intermédiaire des réseaux locaux et régionaux. Mais le grand attachement des Tunisiens à leur pays et la forte cohésion de la société musulmane nous amènent à penser qu'il s'agira d'une évolution lente et que ce champ migratoire continuera à fonctionner encore longtemps». Près de trente ans après, l'hypothèse de la permanence de ce champ migratoire se trouve vérifiée; en dépit de tous les obstacles administratifs élaborés depuis plus de trente ans de législation française, le champ migratoire des Tunisiens existe toujours, ses structures spatiales se sont certes modifiées mais sans perdre leur ossature, leurs structures fondamentales, et il continue à fonctionner efficacement: la circulation des personnes se poursuit, le montant des transferts financiers ne cesse de progresser, les liens personnels, les retours ordinaires en cours d'année n'ont rien perdu de leur vitalité, les liens affectifs semblent encore plus forts qu'autrefois.

Malgré le temps passé, cette formation socio-spatiale est donc toujours en place parce que l'architecture de leur construction sociale était et demeure tout à fait solide. Le paysage migratoire mondial actuel offre de très nombreux exemples de la très grande stabilité dans le temps des champs migratoires, entre pays frontaliers ou positionnés dans des ensembles à très large rayon d'action, la mondialisation migratoire actuelle ne pouvant que développer ce type de structures fonctionnelles. Stabilité ne signifie pas pour autant fixité, rigidité, immobilisme ; le champ migratoire est une structure souple, dynamique susceptible de redéployer sa spatialité vers des espaces beaucoup plus vastes comme le montre l'ouverture des champs migratoires actuels.

La question du fonctionnement des champs migratoires dans la durée, de l'inscription dans le temps long, la capacité de ces cadres spatiaux et territoires transnationaux à durer, leur inertie considérable - ce qui n'induit pas leur fixité -, leur empreinte dans les mentalités collectives, sont telles que leurs structures spatiales peuvent être mobilisées bien longtemps après leur mort apparente et ce, dans le cadre de flux et de logiques fonctionnant à contre-sens, à rebours des champs migratoires initiaux, mais à l'intérieur du même cadre territorial transnational. C'est le cas par exemple des migrations argentines actuelles vers l'Europe qui s'inscrivent dans les cadres des anciens champs migratoires des Italiens et des Espagnols dans ce pays, mais aussi celui des descendants des Japonais vers la mère-patrie et de manière plus générale des mouvements migratoires issus des ex-territoires coloniaux vers les ex-métropoles qui réutilisent - entre autres vecteurs - les cadres spatiaux des champs migratoires issus de la colonisation européenne. La mémoire longue des champs migratoires, mémoire 
culturelle et affective - le champ migratoire n'est pas un espace neutre, affectivement neutre - balise en quelque sorte la géographie des circulations migratoires actuelles.

Ni tout à fait synonymes ou interchangeables, ni opposés et encore moins antagonistes, les concepts de champ migratoire et de territoire circulatoire apportent chacun leur éclairage dans la question complexe des formations spatiales et sociales liées aux mobilités. Chacun a ses qualités génératives de sens, le premier plus axé sur les structures et la puissance évocatoire, le second sur les fonctionnalités et les pratiques des acteurs migrants; chacun a aussi ses limites mais leurs apports théoriques sur le plan de la spatialisation sont importants : universalisme, co-spatialisation de l'analyse migratoire, déclinaison et emboîtement des échelles; approche globale de la dynamique générale du champ et des éléments structurants. Au final, la diversité croissante des termes, la polysémie, le flou lui-même, loin d'être facteurs de trouble et de confusion, nous semblent, au contraire, utiles et même indispensables dans l'approche de la recomposition des espaces de mobilité dans le contexte de la mondialisation actuelle.

\section{BIBLIOGRAPHIE}

ALLEMAND Sylvain, ASCHER François, LEVY Jacques éd. (2004). Les sens du mouvement. Paris. Belin, $336 \mathrm{p}$.

ALMEIDA Castro (1974) Espaces socio-culturels et migrations. Colloque sur les travailleurs immigrés Paris 1974.

BALANDIER Georges (2005) Le Grand Dérangement, Paris, PUF, 119 p.

BÉTEILLE Roger (1974) Les Aveyronnais. Essai géographique sur l'espace humain, Thèse de Doctorat d'État en géographie, Poitiers 573 p.

BRUNEAU Michel (2004) Diasporas et espaces transnationaux. Paris, Anthropos.

COURGEAU Daniel (1970) Les champs migratoires en France. Travaux et Documents. Cahiers de l'INED. $\mathrm{n}^{\circ} 58,160 \mathrm{p}$.

FARET Laurent (2003) Les territoires de la mobilité. Migrations et communautés transnationales entre le Mexique et les États-Unis, Paris, Éditions du CNRS, 351 p.

DI MEO Guy, BULÉON Pascal (2005) L'espace social. Lecture géographique des sociétés, Paris, A. Colin, $303 \mathrm{p}$.

HÄGESTRAND Torsten (1955) Migration and Area. Survey of Swedish Migration Fields and Hypothetical. Considerations on Their Genesis. Migration in Sweden. A symposium. Lund Studies in Geography Ser. B n 13, pp. 27-159.

HILY Marie-Antoinette, MA MUNG Emmanuel (2003) Catégories et lieux de la circulation migratoire, Cahiers de recherches de la MIRE, La Documentation française $n^{\circ}$ 16, pp. 33-39.

KNAFOU Rémy dir (1998) La planète nomade. Les mobilités géographiques d'aujourd'hui. Paris, Belin $247 \mathrm{p}$. 
KRITZ Mary, M., LIN Lean Leam, ZLOTNICK Hania (1992) International Migrations, Systems. A Global Approach, New-York, Oxford University Press, 354 p.

LEVY Jacques (1999) Le tournant géographique. Penser l'espace pour lire le monde, Paris, Belin 1999, $400 \mathrm{p}$.

LEVY Jacques, LUSSAULT Michel éd. (2003) Dictionnaire de la géographie et de l'espace des sociétés, Paris, Belin, $1034 \mathrm{p}$.

MABOGUNJE A.L. (1970) Systems Approach To a Theory of Rural-Urban Migration, Geographical Analysis, vol. 2, pp. 1-17.

MA MUNG Emmanuel (1999) La dispersion comme ressource spatiale, Cultures et conflits $\mathrm{n}^{\circ} 33-34$, pp. 89-103.

Penser l'espace (2005) Séminaire Chôros, Epfl Lausanne www.espaces temps.net/document 1107.

PORTES Alejandro (1999) La mondialisation par le bas. L'émergence des communautés transnationales. Actes de la Recherche en Sciences Sociales n 129, septembre 1999.

ROSENTHAL Paul-André (1999) Les sentiers invisibles. Espaces, familles, migrations dans la France du $X_{X I X}{ }^{e ̀ m e}$ siècle, Éditions de l'École des Hautes Études en Sciences Sociales, 256 p.

SCHWAB Roland (1971) Les champs migratoires : critères d'études de la genèse et de l'évolution des structures régionales de l'espace, Bulletin de l'Association des géographes français, nov-déc, pp. 369-375.

SIMON Gildas (1979) L'espace des travailleurs tunisiens en France. Structures et fonctionnement d'un champ migratoire international. Thèse de Doctorat d'État en géographie, Poitiers, $426 \mathrm{p}$.

SIMON Gildas (1981). Réflexions sur la notion de champ migratoire international, Hommes et Terres du Nord nº spécial, pp. 85-90.

SIMON Gildas (1995) Géodynamique des migrations internationales dans le monde, Paris, PUF, 429 p.

TARRIUS Alain (1989) Anthropologie du mouvement. Paris. Paradigmes 158 p.

TARRIUS Alain (2001) Les nouveaux cosmopolitismes, Éditions de l'Aube, 267 p.

TISSOT Sylvie, POUPEAU Franck La spatialisation des problèmes sociaux, in Politiques des espaces urbains. Penser, classer, administrer la pauvreté (1) Actes de la Recherche en Sciences Sociales $n^{\circ} 159,2005$, pp. 5-9.

URRY John (2005) Sociologie des mobilités. Une nouvelle frontière pour la sociologie ? Paris, Armand Colin, $252 \mathrm{p}$.

\section{RÉSUMÉS}

Dans le changement en profondeur du regard porté sur le domaine des migrations internationales, les questions d'espace, de spatialité, de territorialité ont pris une place croissante à l'intérieur du champ scientifique : rapport des migrants aux lieux et aux espaces matériels, sociaux, politiques ou idéels reliés par ces migrations, pratiques sociales développées à travers des espaces transnationaux, architecture des constructions identitaires à différentes échelles ou encore espaces normatifs ou réglementaires. L'auteur, géographe, s'attache à la spatialisation croissante des modes d'analyse, et faisant un retour sur la notion de champ migratoire, s'interroge sur le sens fort dont ce terme reste porteur dans l'ordre des spatialités, sur la charge tensionnelle et la puissance évocatoire contenues, sur la capacité de ce type de 
structures à se maintenir dans le temps et dans la durée, dans le cadre de la dynamique profonde des espaces de mobilité mondialisés.

Migrations or the spatialization of approaches. Changes in the in-depth look cast upon international migrations, have given increasing importance to issues concerning space, spatiality, territoriality in the scientific field: relationship between migrants and material, social, political or immaterial domains related to those migrations; social practices developed across international space; structure of identity building at various levels or normative or regulatory spaces. The author, a geographer, highlights the increasing spatialization of approaches and, looking back on the notion of migratory field, he questions the strong meaning which that notion retains in connection with spatiality, the level of tension and suggestive potential it still has, the capability of that form of structure to persist over time and in the long term, within the deep dynamic movements of globalized mobility spaces.

Migraciones, la «espacialización» de la visión. En el marco del cambio profundo de la visión de las migraciones internacionales, las cuestiones de espacio, «espacialidad» y territorialidad han ido tomando un lugar creciente en el campo científico : la relación de los inmigrantes a los lugares y a los espacios materiales, sociales, políticos o ideales conectados por estas migraciones; las prácticas sociales desarrolladas a través de espacios transnacionales; la arquitectura de las construcciones de identidad a escalas diferentes o, incluso, los espacios normativos o reglamentarios. El autor, geógrafo, se interesa en la «espacialización» creciente de los modos de análisis y, volviendo a la noción de campo migratorio, se interroga sobre el sentido fuerte que este término conserva en el orden de las «espacialidades», sobre la carga de tensión y la fuerza de evocación contenidas, sobre la capacidad de este tipo de estructuras a mantenerse en el tiempo, en el marco de la dinámica profunda de los espacios de movilidad mundializados.

\section{INDEX}

Mots-clés : recherche, représentations, terminologie, analyse spatiale, circulation migratoire, mobilité géographique

\section{AUTEUR}

\section{GILDAS SIMON}

Professeur émérite de géographie, équipe Migrinter (Unité mixte de recherche 6588 CNRS), Maison des Sciences de l'Homme et de la Société, 99 av du Recteur Pineau, Poitiers, France. Courriel : gilza.simon@wanadoo.fr. 